\title{
Laura Ramello, Un mito alla corte di Borgogna. "Ciperis de Vignevaux" in prosa
}

\section{Maria Colombo Timelli}

\section{(2) OpenEdition}

\section{Journals}

Édition électronique

URL : http://journals.openedition.org/studifrancesi/2096

DOI : 10.4000/studifrancesi.2096

ISSN : 2427-5856

Éditeur

Rosenberg \& Sellier

\section{Édition imprimée}

Date de publication : 1 avril 2014

Pagination : 125-127

ISSN : 0039-2944

\section{Référence électronique}

Maria Colombo Timelli, "Laura Ramello, Un mito alla corte di Borgogna. "Ciperis de Vignevaux" in prosa », Studi Francesi [En ligne], 172 (LVIII | I) | 2014, mis en ligne le 01 avril 2014, consulté le 18 septembre 2020. URL : http://journals.openedition.org/studifrancesi/2096 ; DOI : https://doi.org/10.4000/ studifrancesi.2096

Ce document a été généré automatiquement le 18 septembre 2020.

\section{cc) (†) $\ominus$}

Studi Francesi è distribuita con Licenza Creative Commons Attribuzione - Non commerciale - Non opere derivate 4.0 Internazionale. 


\title{
Laura Ramello, Un mito alla corte di Borgogna. "Ciperis de Vignevaux" in prosa
}

\author{
Maria Colombo Timelli
}

\section{RÉFÉRENCE}

LAURA RAMELLO, Un mito alla corte di Borgogna. "Ciperis de Vignevaux" in prosa, Edizione con note critiche e commento linguistico-letterario, Alessandria, Edizioni dell'Orso, 2012, pp. 129.

1 Longtemps décriées par la critique, les 'mises en prose' des romans et chansons de geste jouissent depuis quelques décennies d'une attention accrue, et leurs éditions se multiplient; c'est dans ce cadre que Laura Ramello publie la réécriture d'un poème épique, Ciperis de Vignevaux, accompagnée d'une traduction italienne en regard. Le texte n'est pas long, et - il faut bien l'admettre - n'est pas un chef-d'œuvre; cependant, on lui reconnaîtra deux mérites: une valeur documentaire, puisque le manuscrit unique de la source en vers est gravement endommagé (on peut évaluer une perte de $50 \%$ du récit), et une valeur de témoignage sur les intérêts du milieu bourguignon où la prose a été rédigée.

2 Le récit, fondé sur l'histoire du héros - neveu du roi Dagobert - et de ses dix-sept enfants, a joui d'un succès relatif: en plus du manuscrit appartenu à Philippe le Bon (aujourd'hui Bruxelles, KBR 3576-3577, la deuxième unité correspondant à la mise en prose du roman Blancandin), on en connaît trois éditions parisiennes du $\mathrm{xvI}^{\mathrm{e}}$ siècle: Veuve de Jean de Saint Denis, Nicolas Chrétien, Jean Bonfons, toutes sans date. Laura Ramello le classe comme un «romanzo storico» (p. 11, plus simplement comme un «romanzo» p. 15), ou encore comme une «biografia cavalleresca pseudo-storica» (p. 14); mais ailleurs elle doit admettre que par son sujet Ciperis appartient au noyau des chansons de geste (p. 13). De fait, comme c'est souvent le cas tant pour les épopées 
tardives que pour les mises en prose à succès, Ciperis associe des éléments nettement romanesques (les amours de Philippe et Clarisse, parents du protagoniste, l'éducation chevaleresque et sentimentale de celui-ci à la cour d'Angleterre) et d'autres certainement épiques (complots multiples, questions politiques, batailles), ce qui ne permet pas vraiment de le classer sur la base de nos étiquettes génériques.

3 Laura Ramello consacre les pages liminaires à des questions littéraires (la réévaluation relativement récente des mises en prose, l'intérêt qu'un récit pseudo-historique à connotation didactique pouvait susciter à la cour de Bourgogne), à l'histoire du texte ( $d u$ poème-source à la version $d u \mathrm{Xv}^{\mathrm{e}}$ siècle), à la langue du remaniement (fortement marquée par des traits picards et du nord-est de la France), au manuscrit, aux critères d'édition. La partie la plus développée, et la plus intéressante, concerne les modifications introduites par le prosateur, qui n'hésite pas à abréger sa source en supprimant - ce qui est commun à bien d'autres 'proses' - les récits de batailles, la plupart des dialogues et des monologues intérieurs, alors qu'il ajoute certains commentaires et introduit des modifications visant à donner plus de cohérence au récit.

4 L'introduction contient quelques inexactitudes qu'il convient de rectifier. Ainsi, on ne peut pas citer, parmi les œuvres composées pour Philippe le Bon ou commanditées par lui, le Livre des faits... de Charles V de Christine de Pizan (sic, p. 11); Laura Ramello parle d'Olivier de Castille comme d'une mise en prose (p. 12); encore, l'article de l'inventaire post mortem de 1467-1468 («Ung livre en papier... quemenchant le second feuillet d'or et d'argent», Barrois n. 1302, cité ici p. 31) est erronément interprété comme une preuve de «la precoce perdita della prima carta». Le caractère acéphale du manuscrit nous prive du prologue, peut-être aussi du titre: à ce propos, l'éditrice a adopté celui de ce même inventaire, ce qui est tout à fait légitime; c'est la motivation qui nous paraît faible: celui-ci «ha ottime probabilità di riflettere l'intitolazione originale» (p. 35). Lorsqu'on connaît à peine le caractère approximatif, sinon fautif, des anciens catalogues, on hésite à souscrire ce genre de justifications.

5 Sans vouloir être trop sévère, signalons aussi les fautes d'impression, assez nombreuses même en italien (un opera, p. 10; mises e prose, p. 10; un'appartenza, p. 12; d'innanzi al quale, p. 19; né è un esempio, p. 26; sbarra obliqua et doppia sbarra, p. 35).

6 La bibliographie citée dans l'Introduction aurait pu être avantageusement mise à jour: Erec (Colombo Timelli 2000), la Reine Berthe (Tylus 2001), la Belle Hélène de Constantinople de Jean Wauquelin (de Crécy 2002) ne sauraient être définies «le ultime edizioni» de mises en prose (note 11 p. 8); les Cent nouvelles nouvelles sont citées dans l'ancienne édition Champion (1928: note 36 p. 12), ce qui est légitime, mais doit être justifié; l'ensemble des manuscrits des mises en prose ont été étudiés récemment par Tania Van Hemelryck («Le livre mis en prose à la cour de Bourgogne. Réflexions pour une approche codicologique d'un phénomène littéraire», in Mettre en prose aux XIV $\mathrm{XVVI}^{\mathrm{e}} e$ siècles, Turnhout, Brepols, 2010, pp. 245-254).

7 C'est surtout sur l'édition que vont porter les remarques qui suivent (dans les renvois, le premier numéro indique la page, le second le paragraphe, selon la numérotation adoptée par Laura Ramello). Le texte de base est celui du manuscrit KBR 3576, complété pour les passages lacunaires par l'édition de la veuve de Jean de Saint Denis, l'apparat critique enregistrant les variantes des deux premières éditions (veuve de Jean de Saint Denis [siglée A] et Nicolas Chrestien [siglée C]): on regrette alors que l'éditrice n'ait pas 
utilisé celles-ci pour amender son texte, lorsqu'il est manifestement fautif. On en donnera quelques exemples.

Pour ce qui est de la transcription, contrairement aux principes énoncés p. 35 («La dieresi è stata posta su $i / y$ in iato»), traïson ne porte pas de tréma $(48 / 4,48 / 6,50 / 4,52 / 5$ etc.); en revanche on le trouve sur hoïr, ce qui est fautif (86/4); qui aurait souvent dû être transcrit qu'i (40/7, 40/12, 42/15, 44/5 (quatre fois), 48/4, 48/5, 54/14 etc.); elle peut correpondre à el le: neantmoins el le pria tant que il luy ouvrit... (40/8); tant el le nourrit qu'i crut mervilleusement (42/15) etc.; il aurait dû être transcrit $i$ l': quant i l'ö̈ ainssi parler (42/16); il lui compta comment i l'avoit trouvé (50/2); comment i l'avoit mis en l'honneur ou il estoit (54/10); Et quant Siperis l'ouyt, i l'en mercia (54/10), etc. Des graphies picardes n'ont sans doute pas été reconnues: ainsi nuitie (44/2), transcrit nuitée; courrie (= courrerie, 62/4), transcrit courrié; de même, abre doit être conservé (58/4), ainsi que sus (= sûrs, 80/5) et Ousaire (100/1) (cf. Gossen, § 56).

9 Comme on l'a dit, le texte de l'édition doit être corrigé à certains endroits: et bailla sa terre a gouvrener, quil le gouvrena bien vaillanment (56/17; lacune, les imprimés donnant $g$. $<a$ ung> qui...; trad.: «e affidò il governo del suo regno, e fu retto molto bene»); Charles Martel se combati a eulx et l'eschaça [lire: les chaça] trestous hors du royalme (60/7, trad.: «e li scacciò tutti»); Lors yssirent hors du chastel et vint atir [imprimés: haster] Yzoré (62/6, trad.: «e Isoré si presentò»); ... et ceulx qui ne le voloient faire se partesissent et s'en alaissent partout bon lui sambleroit (62/7, lire: partout <ou> bon leur s., trad. : «e se ne andassero ovunque avessero voluto»); s'on l'ardoit sa fille, elle se tueroit (64/5, supprimer l'; trad.: «se bruciavano sua figlia...»); il le fist bien mengier, boire bien a son age (66/10; la graphie age pour aise n'est pas attestée dans DMF; trad.: «mangiare e bere a sua volontà»); et le tient on pour saint et nonmé [lire: nonme] saint Gracien (68/2); et Elye disoit a Siperis qu'il se partesist, mais Siperis lui dit qu'il se partesist [souffrist dans les imprimés] encore ung peu (70/4; trad.: «e Elia diceva a Ciperis di voler torneare ma Ciperis gli disse che avrebbe torneato ancora per un po'»); il estoit filz du roy frere Dangobert nonmé Philippe (72/7; du frere $D$. dans les imprimés; trad.: «figlio del fratello di re Dagoberto di nome Filippo»); mais ainchois [le roi] adouba il et fit ainchois [à supprimer] chevalier tous les .xvij filz de Siperis (76/9; trad.: «ma prima addobbò e fece cavalieri...»); et dirent les .iij. autres rois qui [lire : qu'i] lui aideroient a baillier [lire: batillier; imprimés: batailler; trad.: " lo avrebbero aiutato a combattere»]; il arriverent au Tresport ou il avoit une petite fillette (80/5; imprimés: villete; trad.: «dove c'era un priorato»); Lors fut grande la joie <imprimés: quant> l'empereur le sceut (102/4; trad.: «Dunque l'imperatore lo seppe e la gioia fu grande»); ... et leur bailla la lettre que Philippe lui envoioit; lors l'eslut [lire: les lut; lettres est souvent pl. en m.fr.] le roy (102/5).

Un peu plus grave, dans la liste des villes détruites par les ennemis de Charles Martel, la cité de Vermans, et noble c'estoit Arras, et sonme noble c'estoit Amiens (60/7; trad.: «la nobile Arras et la nobilissima Amiens»), Noble et Sonmenoble doivent se lire comme les toponymes anciens accompagnés de leur correspondant en moyen français (à remarquer que les imprimés donnent ici une lectio facilior: «et noble cité d'Arras et la noble cité d'Amiens» A; «noble cité d'Arras et d'Amiens» C). Toujours pour les mots géographiques, Portiugal doit probablement être lu Portingal (94/31); Paine (98/7, «località non identificata» dans la note au texte, p. 99) ne serait pas Pavie?

11 La traduction aussi peut soulever quelques perplexités: plegier qqn dans un duel (= se porter garant pour lui) / patrocinare (48/6, 50/3; mieux: farsi garante per); trouver occasion sur Siperis / «trovare qualche pretesto con Ciperis» (52/7; mieux: contro); une 
damoiselle petite / «una piccola damigella» (52/9; il faut cependant interpréter comme une demoiselle di modesta condizione); mais nulz ne lui en sçavoit dire la verité (68/1: la trad. proposée, «ma l'indagine non diede frutti», comporte un changement de registre peu acceptable); le roy parla a Siperis de pluseurs choses, et meismes $d u$ fait du roy d'Engleterre / «e anche della faccenda del re d'Inghilterra» (74/2; plutôt: e soprattutto); et fist Dagobert crier par tout son rayalme ung tournoy encontre Siperis et ses enfans pour les exauscier (76/10; la fin de la phrase n'est pas traduite: «e fece annunciare... un torneo contro Ciperis e i suoi figli»); et acoeulleerent toutes leur proies et emmenerrent aveux eulx (98/8, trad.: «raccolsero tutte le greggi»; à peine plus bas, 98/9, proies est cependant traduit correctement par «bestiame»).

Pour ce qui est du lexique, en l'absence de glossaire, j'ai relevé quelques locutions qui auraient mérité un petit commentaire, peut-être dans l'Introduction: ce fut une pais fourree (52/5, bien traduit par «fu una pace apparente»), Ciperis leur avoit fait visaige de bos (62/4, trad.: «aveva fatto loro trovare la porta chiusa»), ... et espousa Siperis Orable, et furent les enfans mis soulz le drap (72/10, trad. «e Ciperis sposò Orable, e i figli furono messi sotto il mantello», ce qui ne veut rien dire en italien; par ailleurs, la note à ce passage explique justement que, rapportée à des enfants nés en dehors du mariage, cette expression signifie 'légitimer, reconnaître').

Au total, Laura Ramello met à notre disposition une mise en prose de plus, ce qui ne peut que nous réjouir ; toujours est-il que ce genre d'éditions nous semble mériter le plus grand soin, tant parce que la bibliographie s'est bien enrichie ces dernières années - et mériterait d'être prise en compte pour les lumières que chaque œuvre bien étudiée peut jeter sur le reste de la production - que parce que le moyen français présente des difficultés qu'on ne décèle pas à une première lecture; et encore, parce que, en présence d'un manuscrit unique, on aurait tout à gagner à prendre en compte le reste de la tradition, même lorsque celle-ci est représentée par des imprimés anciens. 